\title{
Single $T$ gate in a Clifford circuit drives transition to universal entanglement spectrum statistics
}

\author{
S. Zhou ${ }^{1}$, Z.-C. Yang ${ }^{1}$, A. Hamma ${ }^{2}$, C. Chamon ${ }^{1}$ \\ 1 Physics Department, Boston University, Boston, MA 02125, USA \\ 2 Physics Department, University of Massachusetts Boston, Boston, MA 02125, USA \\ *zhous@bu.edu
}

April 19, 2020

\begin{abstract}
Clifford circuits are insufficient for universal quantum computation or creating $t$-designs with $t \geq 4$. While the entanglement entropy is not a telltale of this insufficiency, the entanglement spectrum is: the entanglement levels are Poisson-distributed for circuits restricted to the Clifford gate-set, while the levels follow Wigner-Dyson statistics when universal gates are used. In this paper we show, using finite-size scaling analysis of different measures of level spacing statistics, that in the thermodynamic limit, inserting a single $\mathbf{T}$ $(\pi / 8)$ gate in the middle of a random Clifford circuit is sufficient to alter the entanglement spectrum from a Poisson to a Wigner-Dyson distribution.
\end{abstract}

\section{Introduction}

In the past few years, the dynamics of entanglement growth in non-equilibrium settings have been intensively explored, unveiling rich structures and universality classes analogous to equilibrium phenomena [1 5]. Recently, studies along this direction have been extended from entropic measures to the full entanglement spectrum (ES) [6], which captures the finer structure of entanglement. It has been shown that the dynamics of ES is able to distinguish between random unitary circuits of different complexities $[7-9]$, as well as thermalization and localization phases of the underlying Hamiltonian [10 13]. Moreover, the onset of level repulsion in the ES signals the spreading of operator fronts, which serves as an important diagnostic of quantum chaos and information scrambling [14 16].

A crisp example that the ES reflects the complexity of the states generated by a quantum circuit is provided by the analysis of Clifford circuits. These circuits can be efficiently simulated classically and hence are not sufficient for universal quantum computation, due to restricted single-qubit rotations [17,18. Although Clifford circuits can generate states with the same maximal entanglement entropy as Haar random states [19], the ES of such states is either flat (for stabilizer initial states) [4, 20] or Poisson distributed (for random initial product states) 8 as opposed to Wigner-Dyson (W-D) distributed as in the case of Haar random states. Moreover, as shown in [6, 8, the transition between Poisson and $\mathrm{W}-\mathrm{D}$ is connected to the reversibility and learnability of random quantum circuits.

A related important problem is that of derandomization, e.g., phase retrieval and quantum state distinguishability. These tasks require the construction of a $t$-design, that is, a set of gates that reproduces the first $t$ moments of the Haar measure [21]. A random circuit based on the Clifford group can construct a 3 -design but fails to be a 4 -design, which is what one needs for several protocols of derandomization. It is known, though, that Clifford group generates a good approximation of a 4-design 22. In other words, it 

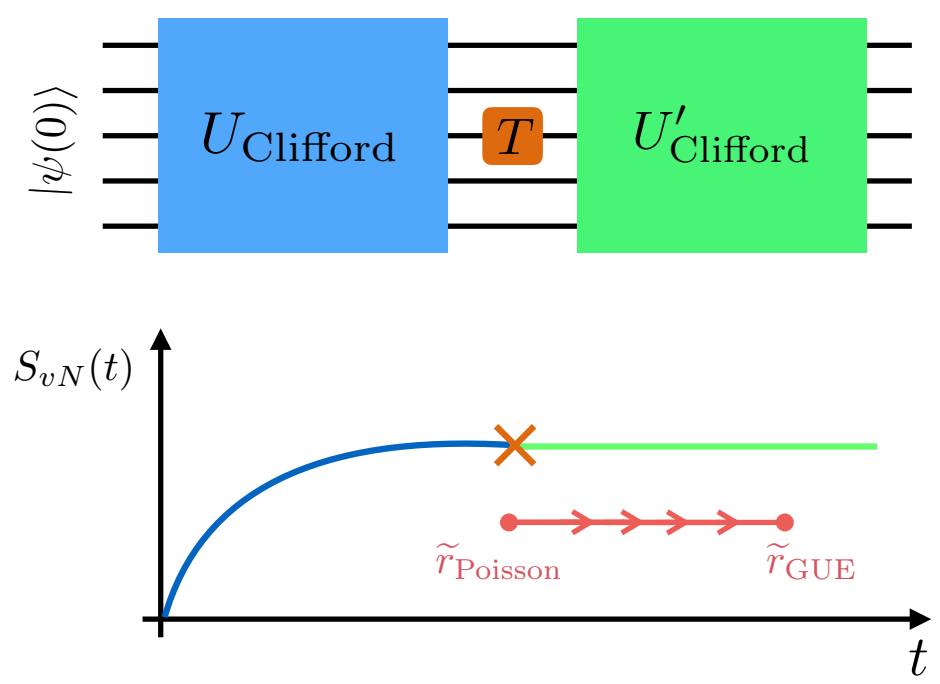

Figure 1: A schematic of the setup considered in this work. Initial product states are first evolved under random Clifford circuits $U_{\mathrm{Cl}}$ until their entanglement entropy reaches maximum. A certain number of $\mathrm{T}$ gates in one layer are then inserted in the circuit, followed by a second stage of random Clifford evolution $U_{\mathrm{Cl}}^{\prime}$. In the thermodynamic limit, the entanglement spectrum flows to Wigner-Dyson distribution upon inserting a single $\mathrm{T}$ gate, and the Poisson fixed point is unstable.

takes very little for the Clifford group to become something that is capable of reproducing the fluctuations of observables evolved with a universal quantum circuit.

In this paper we answer the question of what density of $\mathrm{T}$ gates one needs to add to a Clifford circuit to alter the ES from a Poisson to a W-D distribution, a necessary condition for universal quantum circuits. Moreover, we put forward a conjecture about the transition to unlearnability and higher $t$-designs.

Consider the setup as shown in Fig. 1. We first evolve random product states using random Clifford circuits, until their entanglement entropy reaches maximum. Then we insert a layer of $\mathrm{T}$ gates acting on a certain number of randomly chosen qubits into the circuit, and continue evolving with random Clifford circuits. Since the entanglement entropy has already saturated prior to the insertion of $\mathrm{T}$ gates, it cannot further increase. However, the ES may change following the second stage of time evolution. We ask the question: how many $\mathrm{T}$ gates are needed in the thermodynamic limit to alter the ES from a Poisson to a W-D distribution? Remarkably, we find, using finite-size scaling analysis of various ES statistics measures, that a single $\mathrm{T}$ gate is sufficient to poison the Poisson statistics of pure Clifford circuits in the thermodynamic limit. The deviation from W-D distribution for systems of $N$ qubits scales as $e^{-\gamma n_{T} N}$, where $\gamma$ is a constant of order one and $n_{T}$ is the number of $\mathrm{T}$ gates inserted. This indicates that the ES flows to W-D distribution in the infinite system size limit for any non-zero $n_{T}$. In addition, we also consider two different cases in which either: (1) the initial states are chosen as stabilizer states; or (2) the time evolution following the insertion of $\mathrm{T}$ gates is given by the inverse of the initial evolution. The latter scenario has a natural interpretation in terms of either the noise threshold for reversibility in quantum circuits, or the operator spreading under Clifford dynamics. We find that in the latter case one needs order $\mathcal{O}(\sqrt{N})$ T gates to get W-D distributed ES (for which the density still vanishes as $1 / \sqrt{N}$ ); whereas in the former case, insertion of $\mathrm{T}$ gates in a single layer is not sufficient to obtain $\mathrm{W}$-D distributed ES. 


\section{Setup}

We consider a "composite" quantum circuit consisting of three pieces, as depicted schematically in Fig. 1. The initial state is first evolved under a random Clifford circuit, for which the corresponding unitary operator is denoted as $U_{\mathrm{Cl}}=\prod_{k} U_{k}$, where $U_{k}$ is the evolution operator at $k$-th time step in the circuit. A random Clifford circuit is constructed by picking randomly any of the following three elementary gates with equal probability at each time step: (1) H (Hadamard) gate, which takes $|0\rangle \rightarrow \frac{1}{\sqrt{2}}(|0\rangle+|1\rangle)$ and $|1\rangle \rightarrow \frac{1}{\sqrt{2}}(|0\rangle-|1\rangle)$; (2) $\mathrm{S}(\pi / 4)$ gate, which gives a state-dependent phase factor: $|0\rangle \rightarrow|0\rangle$ and $|1\rangle \rightarrow \mathrm{e}^{i \pi / 4}|1\rangle$; and (3) CNOT (CONTROLLED-NOT) gate, which flips the second qubit conditioned on the state of the first one: $|00\rangle \rightarrow|00\rangle,|01\rangle \rightarrow|01\rangle,|10\rangle \rightarrow|11\rangle,|11\rangle \rightarrow|10\rangle$.

The initial state is evolved for sufficiently long time until the half-system entanglement entropy saturates to its maximal value [19]. We then randomly apply to the state $n_{T} \leq N$ $\mathrm{T}$ gates acting on $n_{T}$ distinct qubits. The $\mathrm{T}$ gate generates a single-qubit rotation about the $\sigma_{z}$-axis similar to the $\mathrm{S}$ gate, but with a different rotation angle: $|0\rangle \rightarrow|0\rangle$ and $|1\rangle \rightarrow \mathrm{e}^{i \pi / 4}|1\rangle$. Remark that replacing $\mathrm{S}$ gate with $\mathrm{T}$ gate leads to a gate set that is sufficient for universal quantum computation [18]. Finally, the state after applying $\mathrm{T}$ gates is further evolved with another random Clifford circuit $U_{\mathrm{Cl}}^{\prime}$ with the same depth as $U_{\mathrm{CL}}$. We shall focus on the case where $U_{\mathrm{Cl}}$ and $U_{\mathrm{Cl}}^{\prime}$ are distinct. However, we also consider in what follows a special situation where $U_{\mathrm{Cl}}^{\prime}=U_{\mathrm{Cl}}^{-1}$. As we will see, the result is quite different in this special case, in contrast to a random $U_{\mathrm{Cl}}^{\prime}$.

The initial states are chosen to be random product states $|\psi(0)\rangle=\otimes_{i=1}^{N}\left|\psi_{i}\right\rangle$, where $\left|\psi_{i}\right\rangle=\cos \theta_{i}|0\rangle+\sin \theta_{i}|1\rangle$ with random angles $\theta_{i} \in[0, \pi]$. Notice that this initial state is not a stabilizer state, and we will briefly comment on the situation of stabilizer initial states at the end. Under random Clifford circuit evolution, the $\operatorname{ES}\left\{p_{k}=\lambda_{k}^{2}\right\}$, defined as the eigenvalues of the reduced density matrix under an equi-bipartitioning of the system $\rho_{A}=\operatorname{tr}_{B}|\psi\rangle\langle\psi|$, exhibits a Poisson level spacing distribution [8, which can be captured by the ratio of adjacent gaps in the spectrum: $r_{k}=\left(\lambda_{k-1}-\lambda_{k}\right) /\left(\lambda_{k}-\lambda_{k+1}\right)$, with $\lambda_{k} \geq \lambda_{k+1}$. Poisson distributed level spacings lead to the following distribution for $r: P(r)=1 /(1+r)^{2}$ with no level repulsion at $r=0$. On the other hand, for levels of random matrix ensembles, the distribution of $r$ follows the W-D surmise 23: $P(r)=\left(r+r^{2}\right)^{\beta} /\left[Z\left(1+r+r^{2}\right)^{1+3 \beta / 2}\right]$, with $Z=\frac{4 \pi}{81 \sqrt{3}}$ and $\beta=2$ for the Gaussian unitary ensemble (GUE).

Since the entanglement entropy is already saturated to its maximum value prior to inserting $\mathrm{T}$ gates, it cannot further increase as a result of subsequent time evolution. Nevertheless, the structure of the state - in particular the ES - could still evolve as the gates realizing $U_{\mathrm{Cl}}^{\prime}$ are sequentially applied. Below, we construct a color map to visualize the wavefunction. This map reveals the change of structure in the bipartite entanglement under the insertion of $\mathrm{T}$ gates. We then extrapolate the evolution of the ES statistics to the thermodynamic limit of $N \rightarrow \infty$ as the number of $\mathrm{T}$ gates varies using numerical simulations and finite-size scaling analysis,

\section{$3 \quad$ Numerics}

We numerically simulate the time evolution protocol of the composite quantum circuit in Fig. 1 for different numbers of $\mathrm{T}$ gates inserted $n_{T} \leq N$. To visualize the structure of a state, we bipartition the system into components $A$ and $B:|\psi\rangle=\sum_{x_{A}, x_{B}} \Psi\left(x_{A}, x_{B}\right)\left|x_{A}\right\rangle\left|x_{B}\right\rangle$, and display $\left|\Psi\left(x_{A}, x_{B}\right)\right|$ by employing a color map with $x_{A}$ in horizontal axis and $x_{B}$ in vertical axis. In Fig. 2, we plot the magnitude of the amplitudes of three states: (1) a state evolved with a Clifford circuit, whose ES is Poisson distributed; (2) a state evolved 

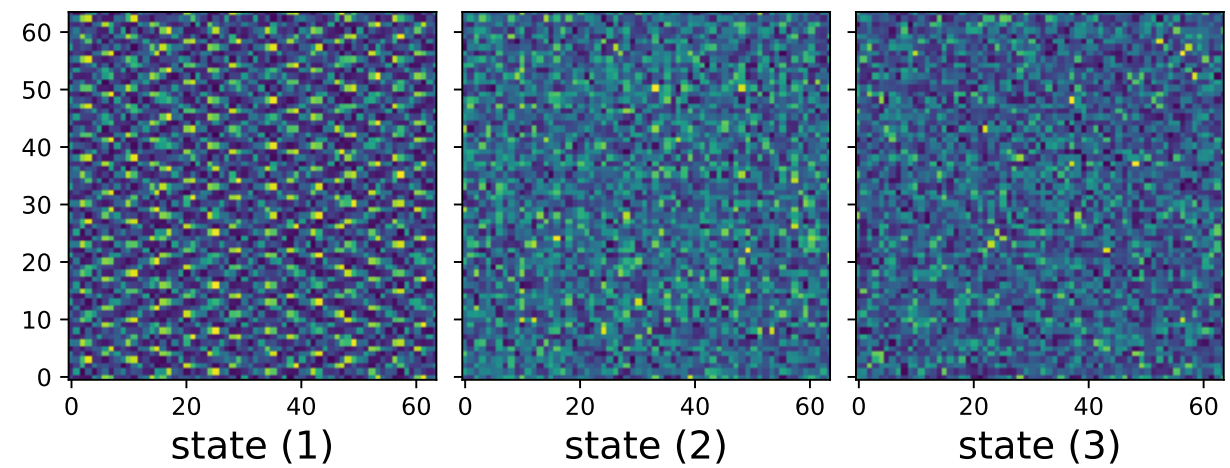

Figure 2: (Color Online) Color Map of the matrix $\Psi\left(x_{A}, x_{B}\right)$, for system size $N=12$, for (1) a state evolved with a Clifford circuit; (2) a state evolved with the composite quantum circuit in Fig. 1 for $12 \mathrm{~T}$ gates; (3) a random state drawn from a Gaussian distribution. Blue (darker) corresponds to lower weights and yellow (lighter) corresponds to higher weights.

with the composite quantum circuit in Fig. 1 for $n_{T}=N$ T gates, whose ES is W-D distributed; (3) a random state with amplitudes drawn from a Gaussian distribution, whose ES is W-D distributed. The existence of a global periodic structure is clearly visible for state (1), but completely absent for the random state (3). The structure in state (1) is a telltale that Clifford circuits cannot fully randomize the state, which is correlated with the absence of level repulsion in the ES. For state (2) with a whole layer of $\mathrm{T}$ gates inserted, we observe no global structure as seen in state (1); instead it resembles the smeared color map shown in state (3), which indicates that adding $\mathrm{T}$ gates into Clifford circuit intrinsically alters Clifford circuit's computational power, and drives the system toward randomness. The striking visual difference between the classical states that can be prepared by action of Clifford gates and quantum states that require universal resources, suggests that such phases can be identified by machine learning architectures based on neural networks [24].

We now can ask the question: how many $\mathrm{T}$ gates are sufficient for a system to realize the transition from a well structured state to a random smeared state. We shall answer below. For the purpose of finite-size scaling, it is favorable to characterize the level spacing distribution of the ES using a single number. We compute a modified version of the $r$-ratio introduced above: $\widetilde{r}_{k}=\min \left\{\delta_{k}, \delta_{k+1}\right\} / \max \left\{\delta_{k}, \delta_{k+1}\right\}$, where $\delta_{k}=\lambda_{k-1}-\lambda_{k}$ is the gap between adjacent eigenvalues 25 . The average value $\langle\widetilde{r}\rangle \approx 0.39$ for Poisson distributed spectrum, and $\langle\widetilde{r}\rangle \approx 0.6$ for GUE distributed spectrum. In Fig. 3 , we plot $\langle\widetilde{r}\rangle$ for for systems of $N=12,14,16,18$ and 20 qubits, and numbers of $\mathrm{T}$ gates inserted. Remarkably, we find that the curves collapse to a universal scaling function of the form:

$$
\langle\widetilde{r}\rangle=f\left(n_{T} \times N\right) .
$$

In other words, $\widetilde{r}$ is only a function of the product $n_{T} N$. One immediately concludes that in the thermodynamic limit, as long as $n_{T} \neq 0,\langle\widetilde{r}\rangle=f(\infty)=\widetilde{r}_{\mathrm{GUE}}$, that is, all that one needs in the thermodynamic limit is a single $\mathrm{T}$ gate to change the ES to random matrix theory behaviors! Moreover, we find that the deviation from $\widetilde{r}_{\mathrm{GUE}}$ has the scaling form:

$$
\delta \widetilde{r} \approx \widetilde{r}_{0} e^{-\gamma n_{T} N},
$$

with $\widetilde{r}_{0}$ a constant, as shown in the inset of Fig. 3 . Eq. (2) implies the following flow equation for $\delta \widetilde{r}$ : $\frac{d \delta \widetilde{r}}{d N} \approx-\gamma n_{T} \delta \widetilde{r}$. The above equations indicate that the Poisson fixed point is unstable, and that the ES in the infinite system size limit will be controlled by 

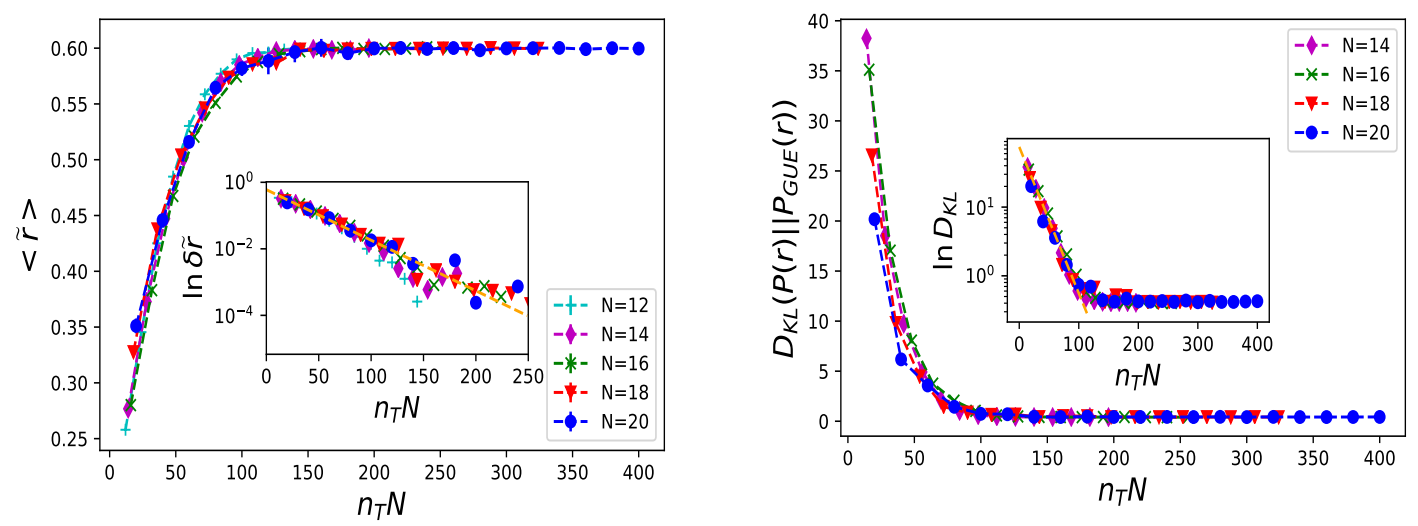

Figure 3: Left: The average $\langle\widetilde{r}\rangle$ defined as $\widetilde{r}_{k}=\min \left\{\delta_{k}, \delta_{k+1}\right\} / \max \left\{\delta_{k}, \delta_{k+1}\right\}$ with $\delta_{k}=\lambda_{k-1}-\lambda_{k}$, versus $n_{T} \times N$, for system sizes $N=10,12,14,16,18$ and 20 . All curves collapse to a universal scaling function $\langle\widetilde{r}\rangle=f\left(n_{T} N\right)$ interpolating between $\widetilde{r}_{\text {Poisson }} \approx 0.39$ and $\widetilde{r}_{\mathrm{GUE}} \approx 0.6$. Inset: deviations from $\widetilde{r}_{\mathrm{GUE}}$ in log-linear scale, indicating the scaling form $\delta \widetilde{r} \approx \widetilde{r}_{0} e^{-\gamma n_{T} N}$. Right:The KL divergence between the full ES level spacing distribution and the W-D distribution. $D_{\mathrm{KL}}$ for different curves also collapses to a universal scaling function of the product $n_{T} N$. Inset: $D_{\mathrm{KL}}$ decays exponentially with $n_{T} N$, similarly to $\delta \widetilde{r}$. The data are averaged over $3000(N=10), 2000(N=12), 1500(N=14), 1000$ $(N=16), 500(N=18)$, and $150(N=20)$ realizations. When not visible, the errorbars are smaller than the size of the data points.

random circuits with $\mathrm{T}$ gates, corresponding to the GUE fixed point. A single $\mathrm{T}$ gate inserted into the Clifford circuit acts like a "poison pill" that completely randomizes the final state and kills the Poisson distribution. This can be understood as follows. Although the density of $\mathrm{T}$ gates is vanishing, it nevertheless changes the global phase structure of the quantum state. The ES is a global property of the full wave function, hence the effect of even a single $\mathrm{T}$ gate is not negligible.

Although the average value of $\langle\widetilde{r}\rangle$ reaches that of GUE in Fig. 3 (left panel), it only characterizes the first moment of the full distribution. To further substantiate that the ES level spacing statistics follows a W-D distribution, we numerically compute the distance between the full distribution of the ES of the final states and the W-D distribution (GUE in particular), defined as the Kullback-Leibler (KL) divergence: $D_{\mathrm{KL}}\left[P(r) \| P_{\mathrm{GUE}}(r)\right]=\sum_{i} P\left(r_{i}\right) \ln \left[P\left(r_{i}\right) / P_{\mathrm{GUE}}\left(r_{i}\right)\right]$. As shown in Fig. 3 (right panel), the KL divergence between the ES level spacing distribution and W-D distribution also collapses to a universal scaling function of the product $n_{T} N$. Therefore, the KL divergence goes to zero in the thermodynamic limit for any nonzero $n_{T}$, confirming that even the full ES level spacing distribution reaches the W-D distribution with the insertion of a single $\mathrm{T}$ gate. The full distribution $P(r)$ corresponding to a particular point where $\langle\widetilde{r}\rangle=\widetilde{r}_{\mathrm{GUE}}$ is presented in Fig. 4, showing that the full distribution of ES level spacing statistics follows W-D.

So far we have been considering the general situation where $U_{\mathrm{Cl}}$ and $U_{\mathrm{Cl}}^{\prime}$ are different and uncorrelated. It is nonetheless interesting to look at a special case in which $U_{\mathrm{Cl}}^{\prime}=U_{\mathrm{Cl}}^{-1}$, i.e the Clifford evolution in the second stage is the exact inverse of the initial evolution operator. This particular example is interesting for several reasons. In the absence of any $\mathrm{T}$ gate inserted in the middle, $U_{\mathrm{Cl}}$ and $U_{\mathrm{Cl}}^{-1}$ will cancel exactly, bringing the final state back to the original product state. However, this cancellation ceases to happen as more and more $\mathrm{T}$ gates are inserted in between. If one views the insertion of $\mathrm{T}$ gates as a particular 


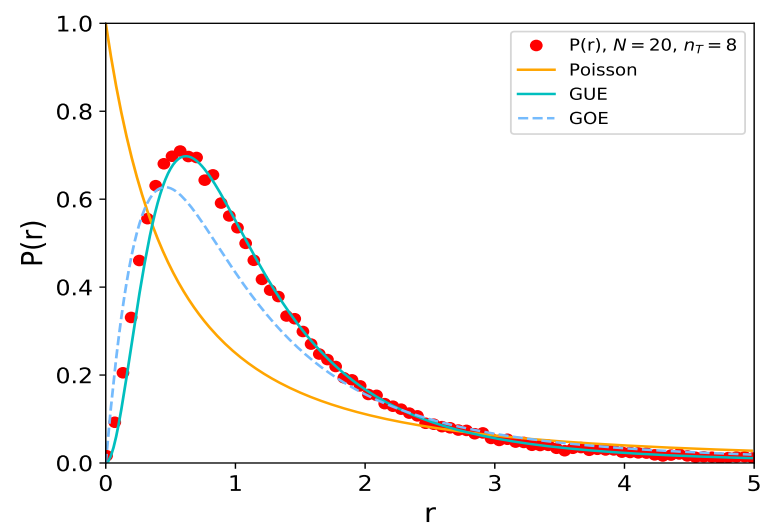

Figure 4: The ES level spacing distribution $P(r)$ for $N=20$ with $n_{T}=8$ T gates inserted.

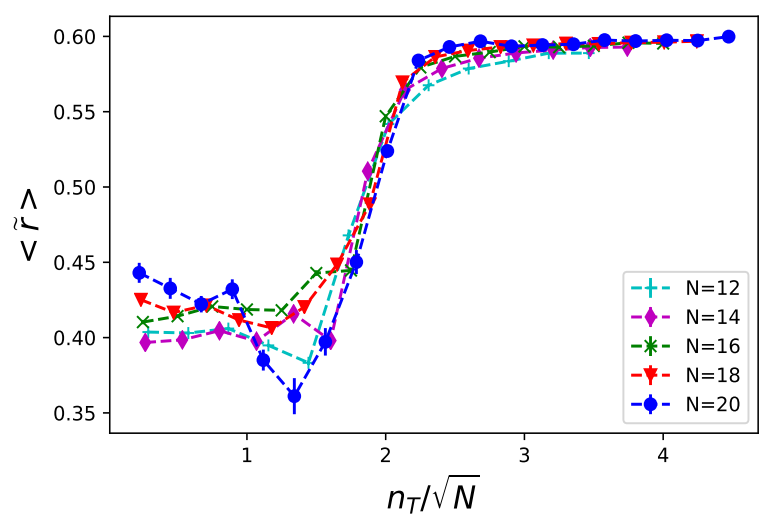

Figure 5: The average $\langle\widetilde{r}\rangle$ versus $n_{T} / \sqrt{N}$ for the special case in which $U_{\mathrm{Cl}}^{\prime}=U_{\mathrm{Cl}}^{-1}$, for systems sizes $N=12,14,16,18$ and 20 . All curves collapse to a universal scaling function $\langle\widetilde{r}\rangle=g\left(n_{T} / \sqrt{N}\right)$ interpolating between $\widetilde{r}_{\text {Poisson }} \approx 0.39$ and $\widetilde{r}_{\text {GUE }} \approx 0.6$. The numbers of realizations are the same as in Fig. 3. When not visible, the errorbars are smaller than the size of the data points.

kind of noise in the circuit, this example gives the noise threshold for reversibility in a quantum circuit. Second, the composite quantum circuit in this particular case coincides with the spreading of $\mathrm{T}$ operators under random Clifford dynamics in the Heisenberg picture. How many $\mathrm{T}$ gates are needed such that their spreading under random Clifford circuit evolution is sufficient to generate W-D distributed ES when acting on a random product state?

In Fig. 5, we plot the average $\langle\widetilde{r}\rangle$ for different system sizes and numbers of $\mathrm{T}$ gates inserted. In contrast to the previous case, here we find instead that different curves collapse to another universal scaling function:

$$
\langle\widetilde{r}\rangle=g\left(n_{T} / \sqrt{N}\right) .
$$

The difference between Eq. (3) and Eq. (1) drastically changes the behavior in the thermodynamic limit. From Fig. 5 , we find that the average $\langle\widetilde{r}\rangle$ reaches $\widetilde{r}_{\text {GUE }}$ when $n_{T} / \sqrt{N} \sim$ $\mathcal{O}(1)$. Therefore, in the infinite system size limit, one needs number $n_{T} \sim \mathcal{O}(\sqrt{N})$ T gates to alter the ES from Poisson to W-D distribution. Notice that even in this case, since $n_{T}$ is only subextensive in sytem size, the required density of T gates still vanishes as $1 / \sqrt{N}$. 
To elucidate how the insertions of the $\mathrm{T}$ gates alter the ES, let us consider explicitly their spreading:

$$
\begin{aligned}
U_{\mathrm{Cl}}\left(\prod_{k=1}^{n_{T}} T_{i_{k}}\right) U_{\mathrm{Cl}}^{-1} & =U_{\mathrm{Cl}}\left[\prod_{k=1}^{n_{T}}\left(\cos \theta \mathbb{1}+i \sin \theta Z_{i_{k}}\right)\right] U_{\mathrm{Cl}}^{-1} \\
& =\prod_{k=1}^{n_{T}}\left(\cos \theta \mathbb{1}+i \sin \theta \widetilde{Z}_{i_{k}}\right)
\end{aligned}
$$

where $\theta=\frac{\pi}{8}$ for the $\mathrm{T}$ gate, and $Z_{i_{k}}$ is the Pauli- $Z$ operator acting on site $i_{k}$. In the second line of Eq. (4), we have used the property that, under Clifford dynamics, a Pauli operator evolves into a single string of Pauli operators $\widetilde{Z}_{i_{k}}$ rather than a superposition of Pauli strings [18]. Upon averaging over circuit realizations, one expects that the $\widetilde{Z}_{i_{k}}$ are essentially random Pauli strings, regardless of the original positions of the inserted T gates. When acting on a product state, each Pauli string operator (and the products of which) $\widetilde{Z}_{i_{k}}$ simply produces another product state, with each qubit being flipped or not depending on the particular type of Pauli operator on each site. Therefore, the time-evolved operator in the above equation will generate a superposition of $2^{n_{T}}$ product states, when applied to a random product state, and the entanglement entropy is thus upper-bounded by $n_{T} \ln 2$. In this situation the insertion of $\mathcal{O}(1)$ T gates is insufficient to discern statistical properties of the ES, since the rank of the density matrix is too low to yield a spectrum with enough non-vanishing eigenvalues. Therefore, when $U_{\mathrm{Cl}}^{\prime}=U_{\mathrm{Cl}}^{-1}$, one needs more than $\mathcal{O}(1) \mathrm{T}$ gates to alter the ES.

In Fig. 6, we plot the ES statistics of states generated by applying the operator in Eq. (4) to random product states, but with the specific $\widetilde{Z}_{i_{k}}$ operator associated with the spreading of $Z_{i_{k}}$ replaced by a random string of Pauli operators. On the left panel, we use $\theta=\frac{\pi}{8}$, corresponding to insertion of T gates, and find that the ES is W-D distributed, in agreement with what was obtained by directly simulating the composite random circuit evolutions. In contrast, if we use $\theta=\frac{\pi}{4}$ instead, corresponding to insertion of $\mathrm{S}$ gates, the ES exhibits a Poisson distribution shown in the right panel, which is consistent with known results for random Clifford circuits [8]. These results indicate that replacing the $\widetilde{Z}_{i_{k}}$ by a random string of Pauli operators in itself does not affect the ES. Instead, it is the angle $\theta$ associated to the specific single-qubit rotation that alters the relative amplitudes of the superposition of $2^{n_{T}}$ product states that affects the ES spectrum. We thus conclude that replacing the specific $\widetilde{Z}_{i_{k}}$ operator associated with $Z_{i_{k}}$ by a random string of Pauli operators has no effect in the ES. Instead, it is the angle $\theta$ associated to the specific singlequbit rotation that alters the relative amplitudes of the superposition of $2^{n_{T}}$ product states to yield the ES associated with the gate set.

We remark that the above results hold when we choose as initial states random product states, where each qubit points along different directions on the Bloch sphere to begin with. Instead, if one starts from stabilizer states, for which random Clifford dynamics yields a flat ES as opposed to Poisson, insertion of T gates in the same manner as in Fig. 11 does not lead to W-D distributed ES. Therefore, the random angles imprinted in the initial states, although by themselves cannot produce Haar random states under Clifford circuit evolution, turns out to be essential for the T gates to wipe off the Poisson distributed ES.

\section{Conclusion}

In this work, we study the critical density of $\mathrm{T}$ gates needed in a random Clifford circuit to alter the ES statistics from Poisson to W-D distribution, a necessary condition for 

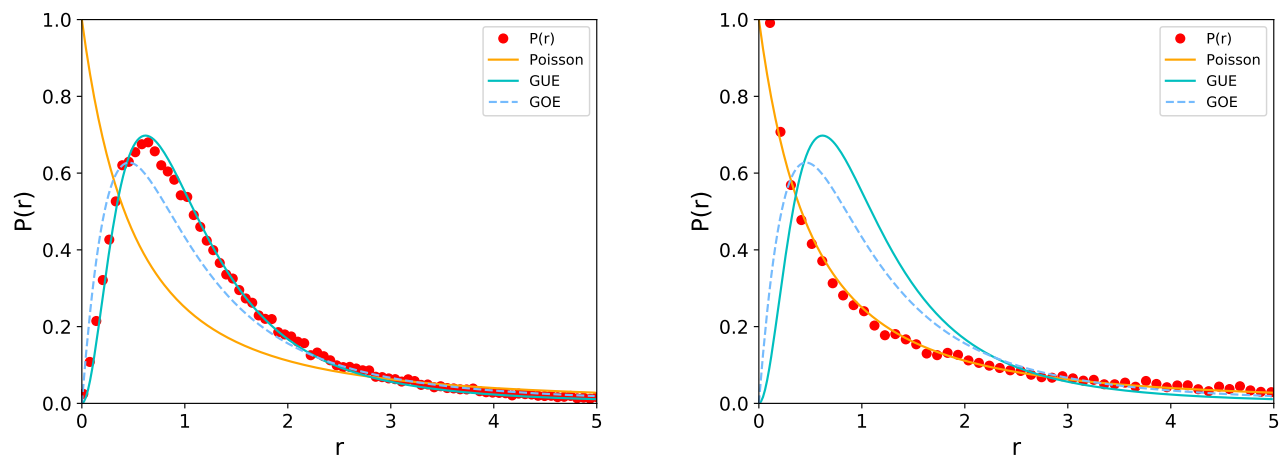

Figure 6: ES statistics of states generated by applying Eq. 4 to random product states. Left: W-D distribution for $\theta=\frac{\pi}{8}$ (inserting T gates); right: Poisson distribution for $\theta=\frac{\pi}{4}$ (inserting $\mathrm{S}$ gates). The data are obtained for system size $N=12$ with $n_{T}=12 \mathrm{~T}$ or $\mathrm{S}$ gate inserted, and averaged over 800 realizations of initial states and random Pauli strings.

the underlying circuit to be universal. We construct a composite quantum circuit as in Fig. 1 with $\mathrm{T}$ gates inserted in the middle, and show that a single $\mathrm{T}$ gate is in fact sufficient to obtain W-D distributed ES, as is the case for Haar random states. Our results suggest that the critical density of $\mathrm{T}$ gates needed for universal quantum computation might be vanishing in the thermodynamic limit. Given the difficulties in realizing T gates within various experimental platforms for quantum computing (e.g. topological quantum computing based on Majorana zero modes), this result points to a new direction where one may compress the non-universal part of the circuit (e.g. the Clifford circuit), while adding only a small number of $\mathrm{T}$ gates at largely spaced layers for universal quantum computation, as shown in Fig. 7. While this may lead to an overhead in the circuit depth, it is advantageous in cases where implementations of $\mathrm{T}$ gates are hard.

In closing, we would like to ask the question of whether the transition driven by the density of non-Clifford gates also characterizes the transition to 4-designs and unlearnability of random quantum circuits. As shown in [6, 8], the transition to W-D for ES level spacing statistics corresponds to the impossibility of reversing the circuit by means of a Metropolis algorithm. This reversal corresponds to learning the circuit, so this is also a learnability-unlearnability transition. We conjecture that learnability is due to the structure of temporal fluctuations in 2-Rényi entropy. This would imply that the transition to the W-D ES is also a transition from 3-design to (at least) 4-design. The Clifford group falls short of being a 4-design but it is a very good approximation of it 22. In particular, it has the same 2-Rényi entropy [26] but not the same fluctuations. We conjecture that the transition to 4-design, W-D and unlearnability are one and the same.

Note Added: The conjecture we put forward in this paper about the critical density of $\mathrm{T}$ gates to drive a Clifford circuit to a 4-design has been recently proved in arxiv:2002.09524.

\section{Acknowledgements}

We thank Thomas Iadecola for useful comments on the manuscript. This work was supported by the U.S. Department of Energy (DOE), Division of Condensed Matter Physics and Materials Science, under Contract No. de-sc0019275. 


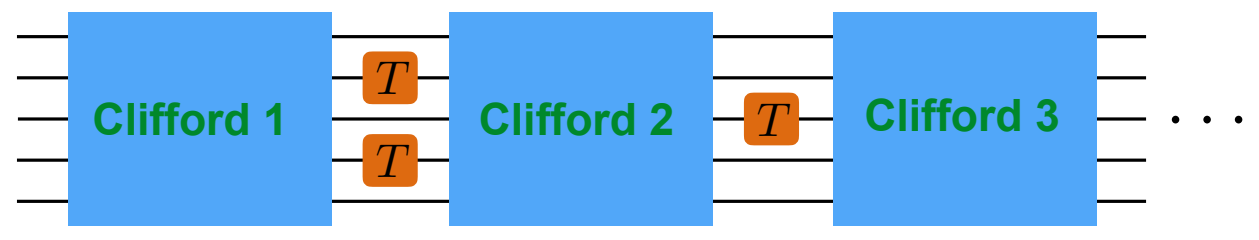

Figure 7: A potential architecture for universal quantum computing, where only a small number of $\mathrm{T}$ gates are added at largely spaced layers.

\section{References}

[1] J. H. Bardarson, F. Pollmann and J. E. Moore, Unbounded growth of entanglement in models of many-body localization, Phys. Rev. Lett. 109, 017202 (2012), doi:10.1103/PhysRevLett.109.017202.

[2] H. Kim and D. A. Huse, Ballistic spreading of entanglement in a diffusive nonintegrable system, Phys. Rev. Lett. 111, 127205 (2013), doi: $10.1103 /$ PhysRevLett.111.127205.

[3] W. W. Ho and D. A. Abanin, Entanglement dynamics in quantum many-body systems, Phys. Rev. B 95, 094302 (2017), doi:10.1103/PhysRevB.95.094302.

[4] A. Nahum, J. Ruhman, S. Vijay and J. Haah, Quantum entanglement growth under random unitary dynamics, Phys. Rev. X 7, 031016 (2017), doi:10.1103/PhysRevX.7.031016.

[5] C. W. von Keyserlingk, T. Rakovszky, F. Pollmann and S. L. Sondhi, Operator hydrodynamics, otocs, and entanglement growth in systems without conservation laws, Phys. Rev. X 8, 021013 (2018), doi:10.1103/PhysRevX.8.021013.

[6] H. Li and F. D. M. Haldane, Entanglement spectrum as a generalization of entanglement entropy: Identification of topological order in non-abelian fractional quantum hall effect states, Phys. Rev. Lett. 101, 010504 (2008), doi:10.1103/PhysRevLett.101.010504.

[7] C. Chamon, A. Hamma and E. R. Mucciolo, Emergent irreversibility and entanglement spectrum statistics, Phys. Rev. Lett. 112, 240501 (2014), doi: $10.1103 /$ PhysRevLett.112.240501.

[8] D. Shaffer, C. Chamon, A. Hamma and E. R. Mucciolo, Irreversibility and entanglement spectrum statistics in quantum circuits, J. Stat. Mech.: Theory and Experiment 2014(12), P12007 (2014).

[9] Z.-C. Yang, K. Meichanetzidis, S. Kourtis and C. Chamon, Scrambling via braiding of nonabelions, Phys. Rev. B 99, 045132 (2019), doi:10.1103/PhysRevB.99.045132.

[10] Z.-C. Yang, C. Chamon, A. Hamma and E. R. Mucciolo, Two-component structure in the entanglement spectrum of highly excited states, Phys. Rev. Lett. 115, 267206 (2015), doi:10.1103/PhysRevLett.115.267206.

[11] S. D. Geraedts, R. Nandkishore and N. Regnault, Many-body localization and thermalization: Insights from the entanglement spectrum, Phys. Rev. B 93, 174202 (2016), doi:10.1103/PhysRevB.93.174202. 
[12] Z.-C. Yang, A. Hamma, S. M. Giampaolo, E. R. Mucciolo and C. Chamon, Entanglement complexity in quantum many-body dynamics, thermalization, and localization, Phys. Rev. B 96, 020408(R) (2017), doi:10.1103/PhysRevB.96.020408.

[13] S. D. Geraedts, N. Regnault and R. M. Nandkishore, Characterizing the many-body localization transition using the entanglement spectrum, New J. Phys. 19(11), 113021 (2017).

[14] X. Chen and A. W. W. Ludwig, Universal spectral correlations in the chaotic wave function and the development of quantum chaos, Phys. Rev. B 98, 064309 (2018), doi:10.1103/PhysRevB.98.064309.

[15] T. Rakovszky, S. Gopalakrishnan, S. Parameswaran and F. Pollmann, Signatures of information scrambling in the dynamics of the entanglement spectrum, arXiv preprint arXiv:1901.04444 (2019).

[16] P.-Y. Chang, X. Chen, S. Gopalakrishnan and J. Pixley, Evolution of entanglement spectra under random unitary dynamics, arXiv preprint arXiv:1811.00029 (2018).

[17] D. Gottesman, The heisenberg representation of quantum computers, arXiv preprint quant-ph/9807006 (1998).

[18] M. A. Nielsen and I. Chuang, Quantum computation and quantum information, Cambridge University Press, Cambridge, UK (2010).

[19] D. N. Page, Average entropy of a subsystem, Phys. Rev. Lett. 71, 1291 (1993), doi:10.1103/PhysRevLett.71.1291.

[20] A. Hamma, R. Ionicioiu and P. Zanardi, Bipartite entanglement and entropic boundary law in lattice spin systems, $\quad$ Phys. Rev. A 71, 022315 (2005), doi:10.1103/PhysRevA.71.022315.

[21] J. Emerson, S. Y. Weinstein, M. Saraceno, S. Lloyd and G. D. Cory, Pseudo-random unitary operators for quantum information processing, Science 302, 2098 (2003), doi:10.1126/science.1090790.

[22] H. Zhu, R. Kueng, M. Grassl and D. Gross, The clifford group fails gracefully to be a unitary 4-design, arXiv preprint arXiv:1609.08172 (2016).

[23] Y. Y. Atas, E. Bogomolny, O. Giraud and G. Roux, Distribution of the ratio of consecutive level spacings in random matrix ensembles, Phys. Rev. Lett. 110, 084101 (2013), doi:10.1103/PhysRevLett.110.084101.

[24] J. Carrasquilla and R. G. Melko, Machine learning phases of matter, Nature Physics p. 431 (2017), doi:10.1038/nphys4035.

[25] V. Oganesyan and D. A. Huse, Localization of interacting fermions at high temperature, Phys. Rev. B 75, 155111 (2007), doi:10.1103/PhysRevB.75.155111.

[26] Z.-W. Liu, S. Lloyd, E. Y. Zhu and H. Zhu, Generalized entanglement entropies of quantum designs, Phys. Rev. Lett. 120, 130502 (2018), doi: $10.1103 /$ PhysRevLett.120.130502. 